\title{
Existence and uniqueness of solutions for fractional boundary value problems with $p$-Laplacian operator
}

\section{Chuanzhi Bai* (B)}

"Correspondence:

czbai8@sohu.com

Department of Mathematics,

Huaiyin Normal University, Huaian,

Jiangsu 223300, P.R. China

\section{Springer}

\begin{abstract}
In this paper, we investigate the existence and uniqueness of solutions for a fractional boundary value problem involving the $p$-Laplacian operator. Our analysis relies on some properties of the Green function and the Guo-Krasnoselskii fixed point theorem and the Banach contraction mapping principle. Two examples are given to illustrate our theoretical results.
\end{abstract}

MSC: 26A33; 34A08; 76F70

Keywords: fractional boundary value problem; $p$-Laplacian operator; fixed point theorem

\section{Introduction}

Fractional differential equations have excited, in the past decades, a considerable interest both in mathematics and in applications. They were used in the mathematical modeling of systems and processes occurring in many engineering and scientific disciplines; for instance, see [1-7]. On the other hand, for studying the turbulent flow in a porous medium, Leibenson [8] introduced the model of a differential equation with the $p$-Laplacian operator. Since then, differential equations with a $p$-Laplacian operator are widely applied in different fields of physics and natural phenomena; for examples, see [9-12] and the references therein.

The topic of fractional-order boundary value problems with the $p$-Laplacian operator has been intensively studied by several researchers in the recent years. We refer the reader to [13-17] and the references therein.

Chen et al. [18] studied the existence of solutions for the boundary value problem of the fractional $p$-Laplacian equation

$$
\left\{\begin{array}{l}
{ }^{c} D_{0^{+}}^{\beta}\left(\varphi_{p}\left({ }^{c} D_{0^{+}}^{\alpha} x(t)\right)\right)=f\left(t, x(t), D_{0^{+}}^{\alpha} x(t)\right), \quad 0<t<1, \\
{ }^{c} D_{0^{+}}^{\alpha} x(0)={ }^{c} D_{0^{+}}^{\alpha} x(1)=0,
\end{array}\right.
$$

where $0<\alpha, \beta \leq 1,1<\alpha+\beta \leq 2,{ }^{c} D_{0^{+}}^{\alpha}$ is the Caputo fractional derivative of order $\alpha$, $\varphi_{p}(s)=|s|^{p-2} s, p>1$, and $f:[0,1] \times \mathbb{R}^{2} \rightarrow \mathbb{R}$ is a continuous function. Obviously, $\varphi_{p}$ is invertible, and its inverse operator is $\varphi_{q}$, where $q>1$ is a constant such that $\frac{1}{p}+\frac{1}{q}=1$.

(c) The Author(s) 2018. This article is distributed under the terms of the Creative Commons Attribution 4.0 International License (http://creativecommons.org/licenses/by/4.0/), which permits unrestricted use, distribution, and reproduction in any medium, provided you give appropriate credit to the original author(s) and the source, provide a link to the Creative Commons license, and indicate if changes were made. 
Arifi et al. [19] investigated the following nonlinear fractional boundary value problem with $p$-Laplacian operator:

$$
\left\{\begin{array}{l}
D_{a^{+}}^{\beta}\left(\varphi_{p}\left(D_{a^{+}}^{\alpha} u(t)\right)\right)+\chi(t) \varphi_{p}(u(t))=0, \quad a<t<b, \\
u(a)=u^{\prime}(a)=u^{\prime}(b)=0, \quad D_{a^{+}}^{\alpha} u(a)=D_{a^{+}}^{\alpha} u(b)=0,
\end{array}\right.
$$

where $2<\alpha \leq 3,1<\beta \leq 2, p>1, D_{a^{+}}^{\alpha}$ is the Riemann-Liouville fractional derivative of order $\alpha$, and $\chi:[a, b] \rightarrow \mathbb{R}$ is a continuous function. Necessary conditions for the existence of nontrivial solutions to (1.2) were given.

In our recent paper [20], we consider the following fractional boundary value problem with mixed fractional derivative and $p$-Laplacian operator

$$
\left\{\begin{array}{l}
D_{a^{+}}^{\beta}\left(\varphi_{p}\left({ }^{c} D_{a^{+}}^{\alpha} u(t)\right)\right)=k(t) f(u(t)), \quad a<t<b, \\
u^{\prime}(a)={ }^{c} D_{a^{+}}^{\alpha} u(a)=0, \quad u(b)={ }^{c} D_{a^{+}}^{\alpha} u(b)=0,
\end{array}\right.
$$

where $1<\alpha, \beta \leq 2, p>1$, and $k:[a, b] \rightarrow \mathbb{R}$ is a continuous function. Under some assumptions on the nonlinear term $f$, the existence of positive solutions to (1.3) was obtained, and two Lyapunov-type inequalities were established.

Motivated by the works mentioned, in this paper, we investigate the existence of positive solutions and the uniqueness of a solution for the following boundary value problem of fractional differential equation with $p$-Laplacian operator:

$$
\left\{\begin{array}{l}
\left(\varphi_{p}\left(D_{0^{+}}^{\alpha} u(t)\right)\right)^{\prime}+f(t, u(t))=0, \quad 0<t<1, \\
u(0)=D_{0^{+}}^{\alpha} u(0)=0, \quad{ }^{c} D_{0^{+}}^{\beta} u(0)={ }^{c} D_{0^{+}}^{\beta} u(1)=0,
\end{array}\right.
$$

where $0<\beta \leq 1,2<\alpha<2+\beta, D_{0^{+}}^{\alpha}$ and ${ }^{c} D_{0^{+}}^{\beta}$ are the Riemann-Liouville fractional derivative and Caputo fractional derivative of orders $\alpha, \beta$, respectively, $p>1$, and $f:[a, b] \times \mathbb{R} \rightarrow \mathbb{R}$ is a continuous function.

The paper is organized as follows. In Section 2, we briefly introduce some necessary basic knowledge and definitions about fractional calculus theory. In Section 3, we write (1.4) as an equivalent integral equation, and then, under some assumptions on the nonlinear $\operatorname{term} f$, we establish three theorems on the existence of nontrivial positive solutions and uniqueness of a solution for FBVP (1.4) by means of the Guo-Krasnoselskii fixed point theorem and the Banach contraction mapping principle, respectively. Finally, in Section 4, we give two examples to show the effectiveness of the results obtained.

\section{Preliminaries}

In this section, we introduce some concepts and results of fractional calculus. For more details, we refer to $[2,3]$.

Definition 2.1 The Riemann-Liouville fractional integral operator of order $\alpha>0$ of a function $f:(0,+\infty) \rightarrow \mathbb{R}$ is given by

$$
\left(I_{0^{+}}^{\alpha} f\right)(t)=\frac{1}{\Gamma(\alpha)} \int_{0}^{t}(t-s)^{\alpha-1} f(s) d s
$$

where $\Gamma$ denotes the gamma function. 
Definition 2.2 The Riemann-Liouville fractional derivative of order $\alpha>0$ of a continuous function $f:(0,+\infty) \rightarrow \mathbb{R}$ is given by

$$
\left(D_{0^{+}}^{\alpha} f\right)(t)=\frac{1}{\Gamma(n-\alpha)} \frac{d^{n}}{d t^{n}} \int_{0}^{t} \frac{f(s)}{(t-s)^{\alpha-n+1}} d s,
$$

where $n=[\alpha]+1$.

Definition 2.3 The Caputo fractional derivative of order $\alpha>0$ of a function $f:(0,+\infty) \rightarrow$ $\mathbb{R}$ is given by

$$
\left({ }^{c} D_{0^{+}}^{\alpha} f\right)(t)=\frac{1}{\Gamma(n-\alpha)} \int_{0}^{t}(t-s)^{n-\alpha-1} f^{(n)}(s) d s,
$$

where $n=[\alpha]+1$.

We now state some properties of fractional operators.

Lemma 2.4 ([3]) Let $\alpha, \beta \in \mathbb{R}^{+}$and $u \in L_{1}[0,1]$. Then $I_{0^{+}}^{\alpha} I_{0^{+}}^{\beta} u(x)=I_{0^{+}}^{\alpha+\beta}$ almost everywhere on $[0,1]$.

Lemma 2.5 ([3]) Let $\alpha \in \mathbb{R}^{+}$and $u \in C[0,1]$. Then ${ }^{c} D_{a^{+}}^{\alpha} I_{a^{+}}^{\alpha} u(x)=u(x)$.

Lemma 2.6 ([3]) If $\alpha>0, n=[\alpha]+1$, and $u \in A C^{n}[0,1]$, then $I_{0^{+}}^{\alpha}{ }^{c} D_{0^{+}}^{\alpha} u(x)=u(x)-$ $\sum_{k=0}^{n-1} \frac{x^{k}}{k !} u^{(k)}(0)$.

Lemma 2.7 ([21]) Let $X$ be a Banach space, and let $P \subset X$ be a cone. Let $\Omega_{1}$ and $\Omega_{2}$ be bounded open subsets of $X$ with $0 \in \Omega_{1} \subset \bar{\Omega}_{1} \subset \Omega_{2}$, and let $T: P \cap\left(\bar{\Omega}_{2} \backslash \Omega_{1}\right) \rightarrow P$ be a completely continuous operator such that

(i) $\|T u\| \geq\|u\|$ for any $u \in P \cap \partial \Omega_{1}$ and $\|T u\| \leq\|u\|$ for any $u \in P \cap \partial \Omega_{2}$; or

(ii) $\|T u\| \leq\|u\|$ for any $u \in P \cap \partial \Omega_{1}$ and $\|T u\| \geq\|u\|$ for any $u \in P \cap \partial \Omega_{2}$.

Then, $T$ has a fixed point in $P \cap\left(\bar{\Omega}_{2} \backslash \Omega_{1}\right)$.

\section{Main results}

Let $E=C[0,1]$ be endowed with the norm $\|x\|=\max _{t \in[0,1]}|x(t)|$.

We now consider the following boundary value problem:

$$
\left\{\begin{array}{l}
\left(\varphi_{p}\left(D_{0^{+}}^{\alpha} u(t)\right)\right)^{\prime}+h(t)=0, \quad 0<t<1, \\
u(0)=D_{0^{+}}^{\alpha} u(0)=0, \quad{ }^{c} D_{0^{+}}^{\beta} u(0)={ }^{c} D_{0^{+}}^{\beta} u(1)=0 .
\end{array}\right.
$$

Lemma 3.1 Let $h \in C[0,1] \cap L[0,1], 0<\beta \leq 1$, and $2<\alpha<2+\beta$. Then $u \in C[0,1]$ is a solution of (3.1) if and only if

$$
u(t)=\int_{0}^{1} G(t, s) \varphi_{q}\left(\int_{0}^{s} h(\tau) d \tau\right) d s,
$$

where $G(t, s)$ is Green's function given by

$$
G(t, s)=\frac{1}{\Gamma(\alpha)} \begin{cases}\frac{(1-s)^{\alpha-\beta-1} t^{\alpha-1}}{\Gamma(\alpha)}, & 0 \leq t \leq s \leq 1 \\ \frac{(1-s)^{\alpha-\beta-1} t^{\alpha-1}-(t-s)^{\alpha-1}}{\Gamma(\alpha)}, & 0 \leq s \leq t \leq 1\end{cases}
$$


Proof Integrating the first equation of $(3.1)$ on $[0, t]$, by the boundary condition $D_{0^{+}}^{\alpha} u(0)=$ 0 we have that

$$
\varphi_{p}\left(D_{0^{+}}^{\alpha} u(t)\right)=\varphi_{p}\left(D_{0^{+}}^{\alpha} u(0)\right)-\int_{0}^{t} h(s) d s=-\int_{0}^{t} h(s) d s
$$

which implies that

$$
D_{0^{+}}^{\alpha} u(t)=-\varphi_{q}\left(\int_{0}^{t} h(s) d s\right)
$$

By Lemma 2.6 we have that

$$
u(t)=-\frac{1}{\Gamma(\alpha)} \int_{0}^{t}(t-s)^{\alpha-1} \varphi_{q}\left(\int_{0}^{s} h(\tau) d \tau\right) d s+c_{1} t^{\alpha-1}+c_{2} t^{\alpha-2}+c_{3} t^{\alpha-3} .
$$

By the boundary value condition $u(0)=0$ we have $c_{3}=0$. Moreover, from Lemmas 2.4 and 2.5 we can easily obtain

$$
\begin{aligned}
{ }^{c} D_{0^{+}}^{\beta} u(t)= & -\frac{1}{\Gamma(\alpha-\beta)} \int_{0}^{t}(t-s)^{\alpha-\beta-1} \varphi_{q}\left(\int_{0}^{s} h(\tau) d \tau\right) d s \\
& +c_{1} \frac{\Gamma(\alpha)}{\Gamma(\alpha-\beta)} t^{\alpha-\beta-1}+c_{2} \frac{\Gamma(\alpha-1)}{\Gamma(\alpha-\beta-1)} t^{\alpha-\beta-2} .
\end{aligned}
$$

By (3.5) and ${ }^{c} D_{0^{+}}^{\beta} u(0)=0$ we have $c_{2}=0$. On the other hand,

$$
0={ }^{c} D_{0^{+}}^{\beta} u(1)=-\frac{1}{\Gamma(\alpha-\beta)} \int_{0}^{1}(1-s)^{\alpha-\beta-1} \varphi_{q}\left(\int_{0}^{s} h(\tau) d \tau\right) d s+c_{1} \frac{\Gamma(\alpha)}{\Gamma(\alpha-\beta)},
$$

which yields that

$$
c_{1}=\frac{1}{\Gamma(\alpha)} \int_{0}^{1}(1-s)^{\alpha-\beta-1} \varphi_{q}\left(\int_{0}^{s} h(\tau) d \tau\right) d s
$$

Substituting $c_{2}=c_{3}=0$ and (3.6) into (3.4), we can obtain that the solution of (3.1) is

$$
u(t)=\int_{0}^{1} G(t, s) \varphi_{q}\left(\int_{0}^{s} h(\tau) d \tau\right) d s
$$

where Green's function $G(t, s)$ is as in (3.3). The proof is completed.

Lemma 3.2 The function $G(t, s)$ has the following properties:

(1) $G(t, s)>0$ for $t, s \in(0,1)$,

(2) $\beta t^{\alpha-1} s(1-s)^{\alpha-\beta-1} \leq \Gamma(\alpha) G(t, s) \leq(\alpha-1) t^{\alpha-2} s(1-s)^{\alpha-\beta-1}$ for $t, s \in[0,1]$.

Proof First, we prove that (1) holds. Since

$$
(1-s)^{\alpha-\beta-1} \geq\left(1-\frac{s}{t}\right)^{\alpha-\beta-1} \geq\left(1-\frac{s}{t}\right)^{\alpha-1}, \quad 0 \leq s \leq t \leq 1,
$$


we have that

$$
(1-s)^{\alpha-\beta-1} t^{\alpha-1} \geq(t-s)^{\alpha-1}
$$

Thus, we easily obtain that $G(t, s)>0$ for $s, t \in(0,1)$.

We now will prove that (2) holds. If $0 \leq s \leq t \leq 1$, then

$$
\begin{aligned}
\Gamma(\alpha) G(t, s) & =(1-s)^{\alpha-\beta-1} t^{\alpha-1}-(t-s)^{\alpha-1} \leq(1-s)^{\alpha-\beta-1}\left[t^{\alpha-1}-(t-s)^{\alpha-1}\right] \\
& =(1-s)^{\alpha-\beta-1}(\alpha-1) \int_{t-s}^{t} x^{\alpha-2} d x \\
& \leq(\alpha-1)(1-s)^{\alpha-\beta-1} t^{\alpha-2}[t-(t-s)] \\
& =(\alpha-1) s(1-s)^{\alpha-\beta-1} t^{\alpha-2} .
\end{aligned}
$$

On the other hand, we have

$$
\Gamma(\alpha) G(t, s) \geq \beta t^{\alpha-1} s(1-s)^{\alpha-\beta-1} .
$$

The proof of (3.8) is the same as that of (2.6) of Lemma 2.7 in [22], and here we omit it. When $0 \leq t \leq s \leq 1$, we get

$$
\begin{aligned}
\Gamma(\alpha) G(t, s) & =(1-s)^{\alpha-\beta-1} t^{\alpha-1} \leq(1-s)^{\alpha-\beta-1} s t^{\alpha-2} \\
& \leq(\alpha-1) t^{\alpha-2} s(1-s)^{\alpha-\beta-1}
\end{aligned}
$$

On the other hand, we have

$$
\Gamma(\alpha) G(t, s)=(1-s)^{\alpha-\beta-1} t^{\alpha-1} \geq \beta t^{\alpha-1} s(1-s)^{\alpha-\beta-1} .
$$

The proof is completed.

Define the cone $P \subset E=C[0,1]$ by

$$
P=\left\{x \in E: x(t) \geq \frac{\beta}{\alpha-1} t^{\alpha-1}\|x\|, \text { for all } t \in[0,1]\right\}
$$

Theorem 3.3 Let $0<\beta \leq 1,2<\alpha<2+\beta$, and $f:[0,1] \times \mathbb{R}_{+} \rightarrow \mathbb{R}_{+}=[0,+\infty)$ be a continuous function. Suppose that there exist two positive constants $r_{2}>r_{1}>0$ such that the following assumptions are satisfied:

(H1) $f(t, x) \geq \rho \varphi_{p}\left(r_{1}\right)$ for $(t, x) \in[0,1] \times\left[0, r_{1}\right]$,

(H2) $f(t, x) \leq \omega \varphi_{p}\left(r_{2}\right)$ for $x \in[0,1] \times\left[0, r_{2}\right]$,

where

$$
\rho=\varphi_{p}\left(\frac{\Gamma(\alpha) 2^{\alpha-1}}{\beta B(q+1, \alpha-\beta)}\right)
$$

and

$$
\omega=\varphi_{p}\left(\frac{\Gamma(\alpha)}{(\alpha-1) B(q+1, \alpha-\beta)}\right) .
$$


Then FBVP (1.4) has at least one nontrivial positive solution $u$ belonging to $E$ such that $r_{1} \leq\|u\| \leq r_{2}$.

Proof From Lemma 3.1 we know that $u \in C[0,1]$ is a solution of (1.4) if and only if $u$ is a solution of the integral equation

$$
u(t)=\int_{0}^{1} G(t, s) \varphi_{q}\left(\int_{0}^{s} f(\tau, u(\tau)) d \tau\right) d s
$$

Let $T: P \rightarrow E$ be the operator defined by

$$
T u(t)=\int_{0}^{1} G(t, s) \varphi_{q}\left(\int_{0}^{s} f(\tau, u(\tau)) d \tau\right) d s
$$

For any $u \in P$, we have by Lemma 3.2 that

$$
\begin{aligned}
T u(t) & \geq \frac{1}{\Gamma(\alpha)} \beta \int_{0}^{1} t^{\alpha-1} s(1-s)^{\alpha-\beta-1} \varphi_{q}\left(\int_{0}^{s} f(\tau, u(\tau)) d \tau\right) d s \\
& =\frac{\beta}{\alpha-1} t^{\alpha-1} \int_{0}^{1} \frac{1}{\Gamma(\alpha)}(\alpha-1) s(1-s)^{\alpha-\beta-1} \varphi_{q}\left(\int_{0}^{s} f(\tau, u(\tau)) d \tau\right) d s \\
& \geq \frac{\beta}{\alpha-1} t^{\alpha-1} \int_{0}^{1} \max _{t \in[0,1]} G(t, s) \varphi_{q}\left(\int_{0}^{s} f(\tau, u(\tau)) d \tau\right) d s \\
& \geq \frac{\beta}{\alpha-1} t^{\alpha-1} \max _{t \in[0,1]} \int_{0}^{1} G(t, s) \varphi_{q}\left(\int_{0}^{s} f(\tau, u(\tau)) d \tau\right) d s \\
& =\frac{\beta}{\alpha-1} t^{\alpha-1}\|T u\|,
\end{aligned}
$$

which implies that $T: P \rightarrow P$. Using the Arzelà-Ascoli theorem, we can prove that $T: P \rightarrow$ $P$ is completely continuous. Let $\Omega_{i}=\left\{u \in P:\|u\| \leq r_{i}\right\}, i=1,2$. From (H1) and Lemmas 3.1 and 3.2 we obtain for $t \in\left[\frac{1}{2}, 1\right]$ and $u \in P \cap \partial \Omega_{1}$ that

$$
\begin{aligned}
(T u)(t) & \geq \int_{0}^{1} \min _{t \in\left[\frac{1}{2}, 1\right]} G(t, s) \varphi_{q}\left(\int_{0}^{s} f(\tau, u(\tau)) d \tau\right) d s \\
& \geq \frac{1}{\Gamma(\alpha)} \int_{0}^{1} \min _{t \in\left[\frac{1}{2}, 1\right]} \beta t^{\alpha-1} s(1-s)^{\alpha-\beta-1} \varphi_{q}\left(\int_{0}^{s} f(\tau, u(\tau)) d \tau\right) d s \\
& =\frac{\beta}{2^{\alpha-1} \Gamma(\alpha)} \int_{0}^{1} s(1-s)^{\alpha-\beta-1} \varphi_{q}\left(\int_{0}^{s} f(\tau, u(\tau)) d \tau\right) d s \\
& \geq \frac{\beta}{2^{\alpha-1} \Gamma(\alpha)} \int_{0}^{1} s(1-s)^{\alpha-\beta-1} \varphi_{q}\left(\int_{0}^{s} \rho \varphi_{p}\left(r_{1}\right) d \tau\right) d s \\
& =\frac{\beta}{2^{\alpha-1} \Gamma(\alpha)} \int_{0}^{1} s(1-s)^{\alpha-\beta-1} \varphi_{q}(s) d s \cdot \varphi_{q}(\rho) r_{1} \\
& =\frac{\beta}{2^{\alpha-1} \Gamma(\alpha)} \int_{0}^{1} s^{q}(1-s)^{\alpha-\beta-1} d s \cdot \varphi_{q}(\rho) r_{1} \\
& =\frac{\beta}{2^{\alpha-1} \Gamma(\alpha)} B(q+1, \alpha-\beta) \cdot \varphi_{q}(\rho) r_{1}=\|u\| .
\end{aligned}
$$


Hence, $\|T u\| \geq\|u\|$ for $u \in P \cap \partial \Omega_{1}$. On the other hand, from (H2) and Lemmas 3.1 and 3.2 we have

$$
\begin{aligned}
\|T u\| & =\max _{t \in[0,1]} \int_{0}^{1} G(t, s) \varphi_{q}\left(\int_{0}^{s} f(\tau, u(\tau)) d \tau\right) d s \\
& \leq \max _{t \in[0,1]} \int_{0}^{1} \frac{\alpha-1}{\Gamma(\alpha)} t^{\alpha-2} s(1-s)^{\alpha-\beta-1} \varphi_{q}\left(\int_{0}^{s} f(\tau, u(\tau)) d \tau\right) d s \\
& =\frac{\alpha-1}{\Gamma(\alpha)} \int_{0}^{1} s(1-s)^{\alpha-\beta-1} \varphi_{q}\left(\int_{0}^{s} f(\tau, u(\tau)) d \tau\right) d s \\
& \leq \frac{\alpha-1}{\Gamma(\alpha)} \int_{0}^{1} s(1-s)^{\alpha-\beta-1} \varphi_{q}\left(\int_{0}^{s} \omega \varphi_{p}\left(r_{2}\right) d \tau\right) d s \\
& =\frac{\alpha-1}{\Gamma(\alpha)} \int_{0}^{1} s(1-s)^{\alpha-\beta-1} \varphi_{q}(s) d s \cdot \varphi_{q}(\omega) r_{2} \\
& =\frac{\alpha-1}{\Gamma(\alpha)} B(q+1, \alpha-\beta) \cdot \varphi_{q}(\omega) r_{2}=\|u\|
\end{aligned}
$$

for $u \in P \cap \partial \Omega_{2}$. Thus, by Lemma 2.7 we have that the operator $T$ has a fixed point in $u \in P \cap\left(\bar{\Omega}_{2} \backslash \Omega_{1}\right)$ with $r_{1} \leq\|u\| \leq r_{2}$, and clearly $u$ is a positive solution for FBVP (1.4). The proof is completed.

Lemma 3.4 ([12]) The $p$-Laplacian operator has the following properties:

(i) If $1<p<2, x y>0$ and $|x|,|y| \geq m>0$, then

$$
\left|\varphi_{p}(x)-\varphi_{p}(y)\right| \leq(p-1) m^{p-2}|x-y| .
$$

(ii) If $p>2$ and $|x|,|y| \leq M$, then

$$
\left|\varphi_{p}(x)-\varphi_{p}(y)\right| \leq(p-1) M^{p-2}|x-y|
$$

Now we are in position to prove the uniqueness of a solution for FBVP (1.4).

Theorem 3.5 Assume that $0<\beta \leq 1,2<\alpha<2+\beta, 1<p<2$, and the following conditions are satisfied:

(H3) For all $r>0$, there exists a nonnegative function $h_{r} \in L[0,1]$ with $0<\int_{0}^{1} h_{r}(t) d t \leq M$ (a positive constant) such that

$$
|f(t, u)| \leq h_{r}(t), \quad \forall(t, u) \in(0,1] \times[-r, r]
$$

(H4) There exists a constant $k>0$ such that

$$
|f(t, u)-f(t, v)| \leq k|u-v|, \quad \forall t \in[0,1], u, v \in \mathbb{R} .
$$

If

$$
0<k<\frac{1}{(\alpha-1)(q-1) M^{q-2} B(3, \alpha-\beta)},
$$

then FBVP (1.4) has a unique solution in $C[0,1]$. 
Proof By (H3) we have that

$$
\left|\int_{0}^{t} f(s, u(s)) d s\right| \leq \int_{0}^{1}|f(s, u(s))| d s \leq \int_{0}^{1} h_{r}(s) d s \leq M, \quad t \in[0,1] .
$$

By Lemmas 3.1 and 3.4(i) and by (3.10) we obtain

$$
\begin{aligned}
|(T u)(t)-(T v)(t)| & \\
\quad & \left|\int_{0}^{1} G(t, s) \varphi_{q}\left(\int_{0}^{s} f(\tau, u(\tau))\right) d \tau-\int_{0}^{1} G(t, s) \varphi_{q}\left(\int_{0}^{s} f(\tau, v(\tau))\right) d s\right| \\
& \leq \int_{0}^{1} G(t, s)\left|\varphi_{q}\left(\int_{0}^{s} f(\tau, u(\tau)) d \tau\right)-\varphi_{q}\left(\int_{0}^{s} f(\tau, v(\tau)) d \tau\right)\right| d s \\
& \leq(q-1) M^{q-2} \int_{0}^{1} G(t, s)\left|\int_{0}^{s} f(\tau, u(\tau)) d \tau-\int_{0}^{s} f(\tau, v(\tau)) d \tau\right| d s \\
& \leq(q-1) M^{q-2} \int_{0}^{1} G(t, s) \int_{0}^{s}|f(\tau, u(\tau))-f(\tau, v(\tau))| d \tau d s \\
& \leq(q-1) M^{q-2} \int_{0}^{1} G(t, s) \int_{0}^{s} k\|u-v\| d \tau d s \\
& \leq(q-1) k M^{q-2}\|u-v\| \int_{0}^{1}(\alpha-1) t^{\alpha-2} s^{2}(1-s)^{\alpha-\beta-1} d s \\
& =(\alpha-1)(q-1) k M^{q-2} t^{\alpha-2} B(3, \alpha-\beta)\|u-v\| \\
& \leq(\alpha-1)(q-1) k M^{q-2} B(3, \alpha-\beta)\|u-v\| \\
& =L_{1}\|u-v\|,
\end{aligned}
$$

where $L_{1}=(\alpha-1)(q-1) k M^{q-2} B(3, \alpha-\beta)$. From condition (3.9) we know that $0<L_{1}<1$. Hence, by means of the Banach contraction mapping principle we obtain that $T$ has a unique fixed point in $E$, that is, that FBVP (1.4) has a unique solution. The proof is completed.

Theorem 3.6 Assume that $0<\beta \leq 1,2<\alpha<2+\beta$, and $p>2$ and that (H4) and the following condition holds:

(H5) There exist constants $\lambda>0$ and $0<\delta<\frac{\alpha-2}{2-q}$ such that

$$
f(t, u) \geq \lambda \delta t^{\delta-1}, \quad \forall(t, u) \in(0,1] \times \mathbb{R}
$$

If

$$
0<k<\frac{1}{(\alpha-1)(q-1) \lambda^{q-2} B(3, \alpha-\beta)},
$$

then FBVP (1.4) has a unique solution in $C[0,1]$.

Proof By (H5) we have

$$
\int_{0}^{t} f(s, u) d s \geq \int_{0}^{t} \lambda \delta s^{\delta-1} d s=\lambda t^{\delta}, \quad \forall(t, u) \in[0,1] \times \mathbb{R}
$$


Obviously, for any $u, v \in E$, we have $|(T u)(0)-(T v)(0)|=0$. For any $t \in(0,1]$, by Lemmas 3.2 and 3.4(ii) and by (3.12) we get

$$
\begin{aligned}
|(T u)(t)-(T v)(t)| & \leq \int_{0}^{1} G(t, s)\left|\varphi_{q}\left(\int_{0}^{s} f(\tau, u(\tau)) d \tau\right)-\varphi_{q}\left(\int_{0}^{s} f(\tau, v(\tau)) d \tau\right)\right| d s \\
& \leq(q-1)\left(\lambda t^{\delta}\right)^{q-2} \int_{0}^{1} G(t, s)\left|\int_{0}^{s} f(\tau, u(\tau)) d \tau-\int_{0}^{s} f(\tau, v(\tau)) d \tau\right| d s \\
& \left.\left.\leq(q-1)\left(\lambda t^{\delta}\right)^{q-2} \int_{0}^{1} G(t, s) \int_{0}^{s} k \mid u(\tau)\right)-v(\tau)\right) \mid d \tau d s \\
& \leq(q-1) k\left(\lambda t^{\delta}\right)^{q-2}\|u-v\| \int_{0}^{1}(\alpha-1) t^{\alpha-2} s^{2}(1-s)^{\alpha-\beta-1} d s \\
& \leq(\alpha-1)(q-1) k\left(\lambda t^{\delta}\right)^{q-2} t^{\alpha-2}\|u-v\| \int_{0}^{1} s^{2}(1-s)^{\alpha-\beta-1} d s \\
& =(\alpha-1)(q-1) k \lambda^{q-2} t^{(q-2) \delta+\alpha-2} B(3, \alpha-\beta)\|u-v\| \\
& \leq(\alpha-1)(q-1) k \lambda^{q-2} B(3, \alpha-\beta)\|u-v\|,
\end{aligned}
$$

which implies that

$$
\|T u-T v\| \leq L_{2}\|u-v\|,
$$

where $L_{2}=(\alpha-1)(q-1) k \lambda^{q-2} B(3, \alpha-\beta)$. By condition (3.11) we obtain that $0<L_{2}<1$. Thus, $T: E \rightarrow E$ is a contraction mapping. Using the Banach contraction mapping principle, we obtain that $T$ has a unique fixed point in $E$. Hence, FBVP (1.4) has a unique solution. The proof is completed.

Similarly, we have the following:

Theorem 3.7 Assume that $0<\beta \leq 1,2<\alpha<2+\beta$, and $p>2$ and that (H4) and the following condition holds:

(H6) There exist constants $\lambda>0$ and $0<\delta<\frac{\alpha-2}{2-q}$ such that

$$
f(t, u) \leq-\lambda \delta t^{\delta-1}, \quad \forall(t, u) \in(0,1] \times \mathbb{R} .
$$

If $k$ satisfies (3.11), then FBVP (1.4) has a unique solution in $C[0,1]$.

\section{Examples}

In this section, we present some examples to illustrate our main results obtained in the previous section.

Example 4.1 We consider the fractional boundary value problem

$$
\left\{\begin{array}{l}
\left|D_{0^{+}}^{\frac{7}{3}} u(t)\right|^{\frac{1}{2}} D_{0^{+}}^{\frac{7}{3}} u(t)+\left(t^{2}+2\right) e^{u}=0, \quad 0<t<1, \\
u(0)=D_{0^{+}}^{\frac{7}{3}} u(0)=0, \quad{ }^{c} D_{0^{+}}^{0.7} u(0)={ }^{c} D_{0^{+}}^{0.7} u(1)=0 .
\end{array}\right.
$$


Obviously, FBVP (4.1) can be regarded as FBVP (1.4) with $p=\frac{5}{2}, \alpha=\frac{7}{3}, \beta=0.7$, and

$$
f(t, u)=\left(t^{2}+2\right) e^{u}, \quad \forall(t, u) \in[0,1] \times \mathbb{R} .
$$

By a simple computation we obtain $q=\frac{5}{3}$,

$$
\rho=\varphi_{\frac{5}{2}}\left(\frac{\Gamma\left(\frac{7}{3}\right) 2^{\frac{4}{3}}}{0.7 \cdot B\left(\frac{8}{3}, \frac{49}{30}\right)}\right)=148.986, \quad \text { and } \quad \omega=\varphi_{\frac{5}{2}}\left(\frac{\Gamma\left(\frac{7}{3}\right)}{\frac{4}{3} \cdot B\left(\frac{8}{3}, \frac{49}{30}\right)}\right)=14.169
$$

We choose $r_{1}=\frac{1}{20}$ and $r_{2}=\frac{2}{3}$. Then we can obtain

$$
\begin{aligned}
& f(t, u)=\left(t^{2}+2\right) e^{u} \geq 2>\rho \varphi_{\frac{5}{2}}\left(r_{1}\right)=1.669 \text { for }(t, u) \in[0,1] \times\left[0, r_{1}\right] \\
& f(t, u)=\left(t^{2}+2\right) e^{u} \leq 3 e^{\frac{2}{3}}<\omega \varphi_{\frac{5}{2}}\left(r_{2}\right)=7.713 \text { for }(t, u) \in[0,1] \times\left[0, r_{2}\right] .
\end{aligned}
$$

Hence, by Theorem 3.3, FBVP (4.1) has at least one nontrivial positive solution $u$ in $E$ such that $\frac{1}{20} \leq\|u\| \leq \frac{2}{3}$.

Example 4.2 Consider the following fractional boundary value problem:

$$
\left\{\begin{array}{l}
\left|D_{0^{+}}^{\frac{5}{2}} u(t)\right|^{-\frac{1}{3}} D_{0^{+}}^{\frac{5}{2}} u(t)+\left(1+2 t^{2}\right) \arctan \left(\frac{2}{3}(u+1)\right)=0, \quad 0<t<1, \\
u(0)=D_{0^{+}}^{\frac{5}{2}} u(0)=0, \quad{ }^{c} D_{0^{+}}^{0.8} u(0)={ }^{c} D_{0^{+}}^{0.8} u(1)=0 .
\end{array}\right.
$$

FBVP (4.2) can be regarded as FBVP (1.4) with $p=\frac{5}{3}, \alpha=\frac{5}{2}, \beta=0.8$, and

$$
f(t, u)=\left(1+2 t^{2}\right) \arctan \left(\frac{2}{3}(u+1)\right), \quad \forall(t, u) \in[0,1] \times \mathbb{R}
$$

It is easy to see that, for any $r>0$,

$$
|f(t, u)| \leq h_{r}(t) \quad \text { for }(t, u) \in[0,1] \times[-r, r]
$$

where $h_{r}(t)=\left(1+2 t^{2}\right) \arctan \left(\frac{2}{3}(r+1)\right)$. Obviously, $h_{r} \in L[0,1]$ and $\int_{0}^{1} h_{r}(s) d s \leq \int_{0}^{1} \frac{\pi}{2}(1+$ $\left.2 s^{2}\right) d s=\frac{5 \pi}{6}:=M$. Moreover, we have

$$
\begin{aligned}
|f(t, u)-f(t, v)| & =\left(1+2 t^{2}\right)\left|\arctan \left(\frac{2}{3}(u+1)\right)-\arctan \left(\frac{2}{3}(v+1)\right)\right| \\
& \leq \frac{2}{3}\left(1+2 t^{2}\right)|u-v| \leq 2|u-v| \quad \text { for } t \in[0,1], u, v \in \mathbb{R} .
\end{aligned}
$$

Let $k=2$. We have

$$
0<k<\frac{1}{(\alpha-1)(q-1) M^{q-2} B(3, \alpha-\beta)}=2.332
$$

where $q=\frac{5}{2}>2$. Thus, by Theorem 3.5, FBVP (4.2) has a unique nontrivial solution. 


\section{Conclusion}

In this paper, we obtain an equivalent integral equation for a class of fractional boundary value problem with $p$-Laplacian operator. Using the properties of the corresponding Green function and Guo-Krasnosel'skii fixed point theorem on cones, we obtain the existence of positive solutions to problem (1.4). Moreover, applying the properties of the $p$-Laplacian operator and the Banach contraction mapping principle, we get some uniqueness results of solutions. Finally, we provide two examples to illustrate the main results.

\section{Acknowledgements}

The author thanks the editor and referees for their careful reading of the manuscript and a number of excellent suggestions.

\section{Funding}

This work is supported by Natural Science Foundation of China (11271364, 10771212).

\section{Competing interests}

The author declares that he has no competing interests.

\section{Authors' contributions}

The author read and approved the final manuscript.

\section{Publisher's Note}

Springer Nature remains neutral with regard to jurisdictional claims in published maps and institutional affiliations.

Received: 10 September 2017 Accepted: 28 December 2017 Published online: 10 January 2018

\section{References}

1. Miller, KS, Ross, B: An Introduction to the Fractional Calculus and Fractional Differential Equations. Wiley, New York (1993)

2. Podlubny, I: Fractional Differential Equations, Academic Press, San Diego (1999)

3. Kilbas, AA, Srivastava, HM, Trujillo, JJ: Theory and Applications of Fractional Differential Equations. Elsevier, Amsterdam (2006)

4. Lakshmikantham, V, Leela, S, Vasundhara, V: Theory of Fractional Dynamic Systems. Cambridge Academic Publishers, Cambridge (2009)

5. Debnath, L, Bhatta, D: Solutions to few linear fractional inhomogeneous partial differential equations in fluid mechanics. Fract. Calc. Appl. Anal. 7, 21-36 (2004)

6. Chen, W, Sun, H, Zhang, X, Korosak, D: Anomalous diffusion modeling by fractal and fractional derivatives. Comput. Math. Appl. 59, 1754-1758 (2010)

7. Paola, M, Pinnola, F, Zingales, M: Fractional differential equations and related exact mechanical models. Comput. Math. Appl. 66, 608-620 (2013)

8. Leibenson, LS: General problem of the movement of a compressible fluid in a porous medium. Izv. Akad. Nauk Kirg. SSR 9, 7-10 (1983) (in Russian)

9. Chen, T, Liu, W, Yang, C: Antiperiodic solutions for Liénard-type differential equation with p-Laplacian operator. Bound. Value Probl. 2010, Article ID 194824 (2010)

10. Jiang, D, Gao, W: Upper and lower solution method and a singular boundary value problem for the one-dimensional p-Laplacian. J. Math. Anal. Appl. 252, 631-648 (2000)

11. Zhang, $X, L i u, L, W u, Y$ : The uniqueness of positive solution for a fractional order model of turbulent flow in a porous medium. Appl. Math. Lett. 37, 26-33 (2014)

12. Liu, X, Jia, M, Xiang, X: On the solvability of a fractional differential equation model involving the $p$-Laplacian operator. Comput. Math. Appl. 64, 3267-3275 (2012)

13. Lu, L, Han, L, Sun, R, Liu, J: Existence on positive solutions for boundary value problems of nonlinear fractional differential equations with p-Laplacian. Adv. Differ. Equ. 2013, 30 (2013)

14. Han, Z, Lu, H, Zhang, C: Positive solutions for eigenvalue problems of fractional differential equation with generalized p-Laplacian. Appl. Math. Comput. 257, 526-536 (2015)

15. Ding, Y, Wei, Z, Xu, J, O'Regan, D: Extremal solutions for nonlinear fractional boundary value problems with p-Laplacian. J. Comput. Appl. Math. 288, 151-158 (2015)

16. Li, Y, Yang, H: Existence of positive solutions for nonlinear four-point Caputo fractional differential equation with p-Laplacian. Bound. Value Probl. 2017, 75 (2017)

17. Liu, X, Jia, M, Ge, W: The method of lower and upper solutions for mixed fractional four-point boundary value problem with p-Laplacian operator. Appl. Math. Lett. 65, 56-62 (2017)

18. Chen, T, Liu, W, Hu, Z: A boundary value problem for fractional differential equation with $p$-Laplacian operator at resonance. Nonlinear Anal. 75, 3210-3217 (2012)

19. Arifi, NA, Altun, I, Jleli, M, Lashin, A, Samet, B: Lyapunov-type inequalities for a fractional $p$-Laplacian equation. J. Inequal. Appl. 2016, 189 (2016)

20. Liu, Y, Xie, D, Yang, D, Bai, C: Two generalized Lyapunov-type inequalities for a fractional $p$-Laplacian equation with fractional boundary conditions. J. Inequal. Appl. 2017, 98 (2017) 
21. Guo, D, Lakshmikantham, V: Nonlinear Problems in Abstract Cones. Notes and Reports in Mathematics in Science and Engineering, vol. 5. Academic Press, Boston (1988)

22. Wang, Y, Liu, L, Wu, Y: Positive solutions for a fractional boundary value problem with changing sign nonlinearity. Abstr. Appl. Anal. 2012, Article ID 214042 (2012)

Submit your manuscript to a SpringerOpen ${ }^{\circ}$ journal and benefit from:

- Convenient online submission

- Rigorous peer review

- Open access: articles freely available online

- High visibility within the field

- Retaining the copyright to your article

Submit your next manuscript at $\gg$ springeropen.com 